\title{
Circular RNA hsa_circ_009755 downregulation correlates with clinicopathology in oral squamous cell carcinoma
}

This article was published in the following Dove Press journal: OncoTargets and Therapy

\author{
Zihong Wang ${ }^{1,2, *}$ \\ Jing Tang ${ }^{3, *}$ \\ Yufan Wang \\ Shuai Sun' \\ Yuling Chen' \\ Yuehong Shen' \\ Hong-Yu Yang' \\ 'Department of Oral and Maxillofacial \\ Surgery, Peking University Shenzhen \\ Hospital, Shenzhen, Guangdong, People's \\ Republic of China; ${ }^{2}$ Peking University \\ Shenzhen Hospital Clinical College, \\ Guangzhou Medical University, \\ Guangzhou, Guangdong, People's \\ Republic of China; ${ }^{3}$ Jingzhou Central \\ Hospital, Jingzhou, Hubei, People's \\ Republic of China \\ *These authors contributed equally to \\ this work
}

Background: Oral squamous cell carcinoma (OSCC) is the most frequent oral malignancy in humans. Increasing numbers of studies suggest that circular RNAs (circRNAs) play a major role in tumorigenesis and tumor progression.

Purpose: We aimed to identify differently expressed circRNA in OSCC and adjacent normal tissue. As well as to study the correlation of circRNA expression with clinicopathological feature in patients with OSCC.

Patients and methods: Primary OSCC tissues and adjacent normal tissues from twentyseven OSCC patients were collected. The expression level of hsa_circ_009755 were analyzed by qRT-PCR.

Results: The measurement results from qRT-PCR revealed that hsa_circ_009755 expression was low in OSCC tissue samples and cell lines, and the low expression of hsa_circ_009755 was closely related to a clinicopathological feature in patients with OSCC. Moreover, the area under the receiver operating characteristic curve was 0.782 , which indicates the sensitivity and specificity of the diagnostic value.

Conclusion: Our data indicates that hsa_circ_009755 could serve as a potential biomarker for OSCC diagnosis.

Keywords: oral squamous cell carcinoma, circular RNA, biomarker, hsa_circ_009755

\section{Introduction}

Oral cancer is one of the 10 most common cancers worldwide. ${ }^{1}$ The cancer sites in the oral cavity include the buccal mucosa, floor of the mouth, anterior tongue, alveolar ridge, retromolar trigone, and hard palate. ${ }^{2}$ The tumors that develop affect basic physiological functions such as chewing, ability to swallow and breathe, sensing, vision, and hearing. ${ }^{2}$ Therefore, halting disease progression in patients with oral cancer is extremely critical. Oral cancer is a malignant tumor that has traditionally been defined as oral squamous cell carcinoma (OSCC), and $\sim 90 \%$ of the cancers are histologically found to originate in squamous cells. ${ }^{1}$ The prognosis for patients with OSCC has not improved markedly despite therapeutic advances in the case of this cancer and several other malignancies, and the 5-year survival rate from OSCC has increased only modestly in the past 30 years and remains at $\sim 55-60 \%{ }^{3}$ Therefore, identification of novel therapeutic targets is crucial for OSCC treatment, and to achieve this goal, it is essential to enhance our understanding of the molecular mechanisms underlying OSCC.
Correspondence: Yuehong Shen; Hong-Yu Yang

Department of Oral and Maxillofacial Surgery, Peking University Shenzhen Hospital, No. I I 20 Lianhua Road, Shenzhen, Guangdong 51800I, People's Republic of China

Tel +8675583923333 ext 72II

Email yuehongshen@hotmail.com hyyang192@hotmail.com 
The advent of next-generation sequencing has led to an increasingly rapid growth of the catalog of identified DNA and RNA molecules. Circular RNAs (circRNAs), which feature covalently linked $3^{\prime}$ and $5^{\prime}$ ends, constitute a class of RNA recently discovered to be stable and abundant, ${ }^{4,5}$ and circRNAs not only extensively regulate cellular activities, but also correlate with oncogenesis and/or tumor progression and are implicated in diverse cancers, such as gastric cancer, breast cancer, esophageal squamous cell carcinoma, and hepatocellular carcinoma. $^{6-9}$ Although circRNAs thus hold the potential to serve as novel biomarkers, little is known regarding the role of circRNAs in OSCC.

By using high-throughput sequencing, microarray of circRNA expression profiles was acquired from patients with OSCCs $(n=8)$ and adjacent normal tissue controls $(n=8)$. We found that hsa_circ_009755 are downregualted in OSCC. Moreover, low-level expression of the circRNA hsa circ_009755 was associated with I-II T stage $(P=0.011)$ in patients with OSCC. Thus, we used a receiver operating characteristic (ROC) curve to evaluate the diagnostic value of hsa_circ_009755, and our data indicate that hsa_circ_009755 could represent a promising biomarker for OSCC diagnosis.

\section{Materials and methods}

\section{Patients and specimens}

All primary OSCC tissues and adjacent normal tissues were collected from patients who had undergone surgery at the Department of Oral and Maxillofacial Surgery, Shenzhen Hospital, Peking University (Shenzhen, China), between December 2016 and May 2017. All samples were confirmed by experienced pathologists. The clinical and pathological characteristics of each patient were collected after surgery. Tissue samples were frozen in liquid nitrogen and stored at $-80^{\circ} \mathrm{C}$. All patients received written informed consent, which was approved by the Clinical Research Ethics Committee of Peking University Health Science Center (IRB0000105308043). The study was designed and conducted in accordance with the principle of the Declaration of Helsinki.

\section{RNA extraction}

Total RNA was isolated from OSCC tissues and adjacent normal tissues by using TRIzol reagent (Invitrogen, Carlsbad, CA, USA) according to the manufacturer's protocol, and the concentration of the RNA samples was measured using a NanoDrop ND2000 spectrophotometer (NanoDrop, Wilmington, DE, USA). Quantitative RT-PCR (qRT-PCR) analysis of circRNAs and $\beta$-actin gene was performed on an ABI PRISM7500 Sequence Detection System (Applied Biosystems, Life Technologies, Waltham, MA, USA) by using a PrimeScript RT Reagent Kit (Takara Bio, Nojihigashi, Kusatsu, Japan) and SYBRGreen Premix Ex Taq (Takara Bio) as per manufacturer's instructions. The sequences of the primers used were the following: hsa_circ_009755, 5'-TGCAGCTCTGGAAAT GCCAAT-3' (forward) and 5'-TGGTCACGTCACACAG AAATGAA-3' (reverse); $\beta$-actin, 5'-AAACTGGAACG TTGAGAGTG-3' (forward) and 5'-AGTGGTCTGGCTT TTAGGT-3' (reverse). The obtained data were analyzed using the $\Delta \mathrm{Ct}$ method. ${ }^{10}$ Data were plotted as cycle threshold value ( $\mathrm{Ct}$ value), in which the $\mathrm{Ct}$ value obtained from the intensity of the fluorescence. The Ct of target mRNA was normalized by the $\mathrm{Ct}$ of the reference gene $\beta$-actin and recorded as $\Delta \mathrm{Ct}$ of each sample. $\Delta \mathrm{Ct}$ shows the difference of expression between two genes ( $\mathrm{Ct}$ of target gene - $\mathrm{Ct}$ reference gene). Therefore, higher $\Delta \mathrm{Ct}$ values indicate lower concentrations of the target gene templates and the lower expressions of the corresponding target genes.

\section{Cell culture}

Three human OSCC cell lines, SCC15, SCC25, and CAL27, were purchased from the College of Stomatology, Wuhan University (Wuhan, China). Human oral keratinocyte (HOK) cells were obtained from the Cell Bank of the Chinese Academy of Sciences (Shanghai, China), where the cells were authenticated based on mycoplasma detection. The cell lines used have been approved by the Clinical Research Ethics Committee of Peking University Health Science Center. SCC15, SCC25, CAL27, and HOK cells were maintained in DMEM (Gibco, Waltham, MA, USA) supplemented with $10 \% \mathrm{FBS}$ and $50 \mathrm{U} / \mathrm{mL}$ penicillin and streptomycin sulfate. All cells were cultured at $37^{\circ} \mathrm{C}$ in $5 \% \mathrm{CO}_{2}$.

\section{Prediction of the circRNA/miRNA/ mRNA interaction}

The miRNA-binding sites were predicted by Mireap, Miranda (v3.3a), and TargetScan (Version:7.0). The targeted miRNAs and mRNAs were predicted by miRTarBase (v6.1).

\section{Statistical analysis}

Statistical analyses were performed using GraphPad Prism 5.0 Software for Windows (GraphPad Software, La Jolla, CA, USA) and Service Solutions SPSS software 16.0 (SPSS, Chicago, IL, USA). Differences in the levels of hsa_circ_009755 between OSCC tissues and paired 
adjacent normal tissues and between OSCC cell lines and HOK cells were assessed using $t$-tests for paired data. One-way ANOVA was used to examine the correlation between hsa_circ_009755 levels and clinicopathological factors, and the ROC curve was established to evaluate diagnostic values. $P<0.05$ was considered statistically significant.

\section{Results \\ Low expression level of hsa_circ_009755 in OSCC tissues}

By using high-throughput screening of circRNA microarrays, we found that hsa_circ_009755 was downregulated in OSCC tissues (Figure 1). Furthermore, we confirmed that hsa_circ_009755 is encoded from chr6 $(82,169,983-82,247,754)$. Featuring a spliced sequence length of 305 bp, hsa_circ_009755 (associated gene symbol IBTK) is composed of 4 exons (exons 13-16) (Figure 2). By performing RNA-seq analyses, we determined the expression level of hsa_circ_009755 in each pair of cancer tissue and adjacent nontumorous tissue; hsa_circ_009755 was expressed at significantly lower levels in OSCC tissues than in the corresponding nontumorous tissues $(\mathrm{n}=27, P<0.001)$ (Figure 3$)$.

\section{Low-level expression of hsa_circ_009755 in OSCC cell lines}

We next measured hsa_circ_009755 expression levels in a normal cell line (HOK) and in 3 human OSCC cell lines, CAL27, SCC15, and SCC25. The expression levels in the tumor cell lines were considerably lower than that in the normal cell line (Figure 4).

\section{Correlation between clinicopathological features and hsa_circ_009755 expression levels}

The association of hsa_circ_009755 expression with several clinicopathological features of OSCC patients was analyzed (Table 1): We found that hsa_circ_009755 levels were related to $\mathrm{T}$ stage $(P=0.011)$, but we did not find association between hsa_circ_009755 levels and other clinicopathological factors (age, gender, tumor differentiation, tumor site, and lymphatic metastasis; $P>0.05$ ).

\section{Diagnostic value of hsa_circ_009755 in OSCC}

To estimate the diagnostic value of hsa_circ_009755 in OSCC, an ROC curve was constructed for differentiating

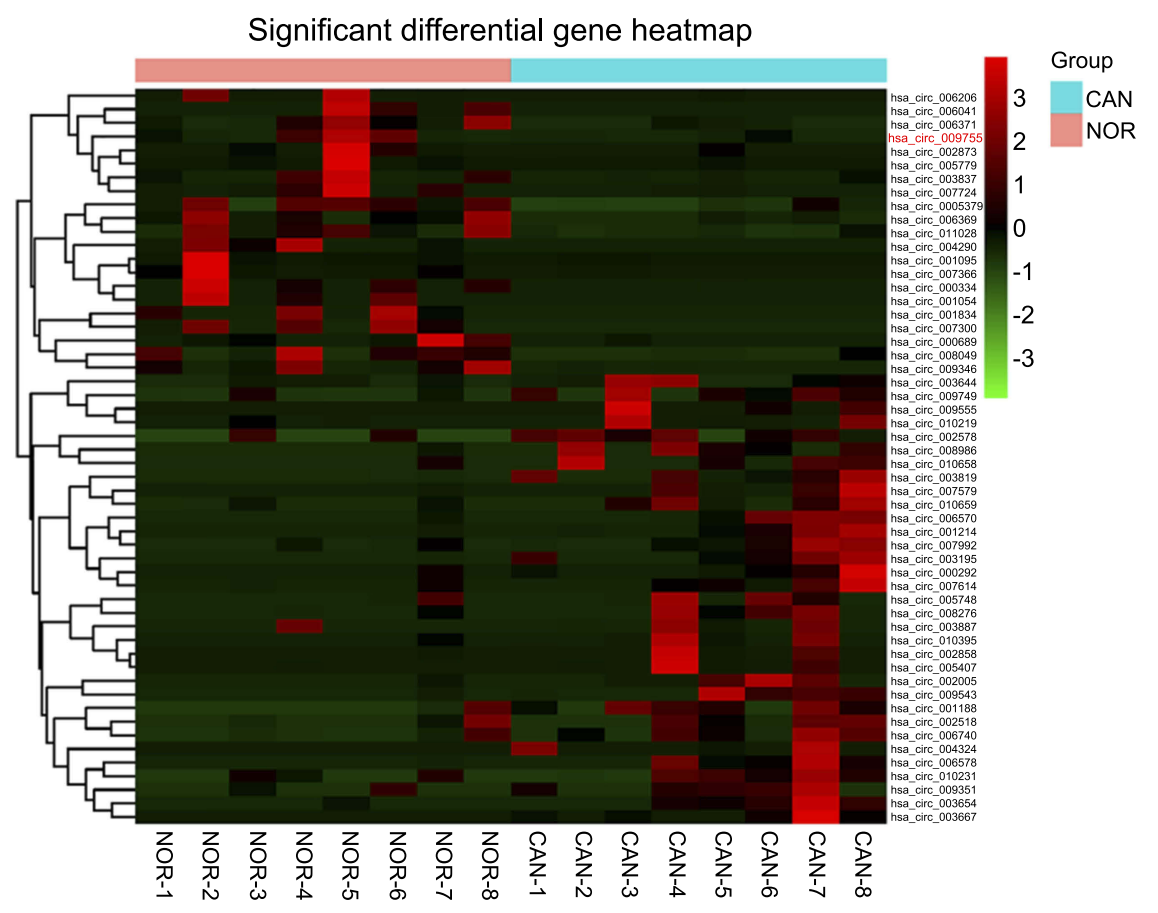

Figure I Differentially expressed circRNAs between OSCC tissues and adjacent nontumorous tissues. The results of hierarchical cluster analysis showed that the differentiable expression profiles of circRNA between the samples (for OSCC, CAN, and NOR). Each column represents the expression of tissue samples. The expression level was indicated from "red" (high expression) to "green" (low expression).

Abbreviations: CAN, cancerous; NOR, normal adjacent nontumorous tissue; OSCC, oral squamous cell carcinoma. 


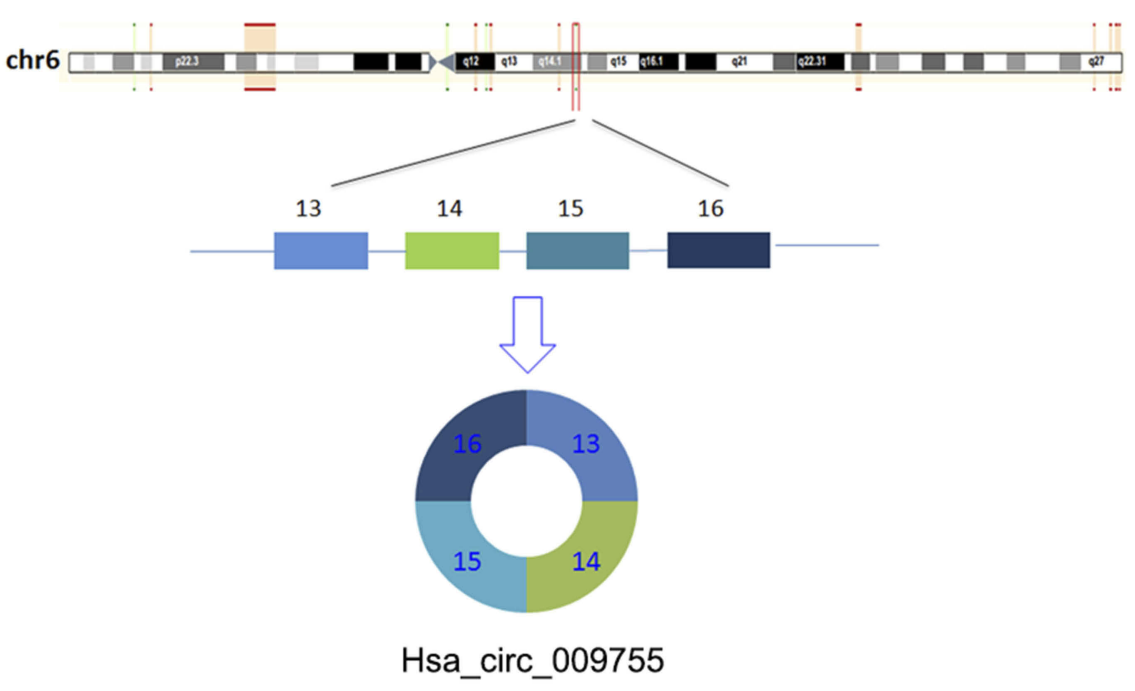

Figure 2 Characterization of hsa_circ_009755. The circRNA hsa_circ_009755 is encoded from chromosomal region 6ql4.I. Four exons in this region (exons I3-16) generate hsa_circ_009755.

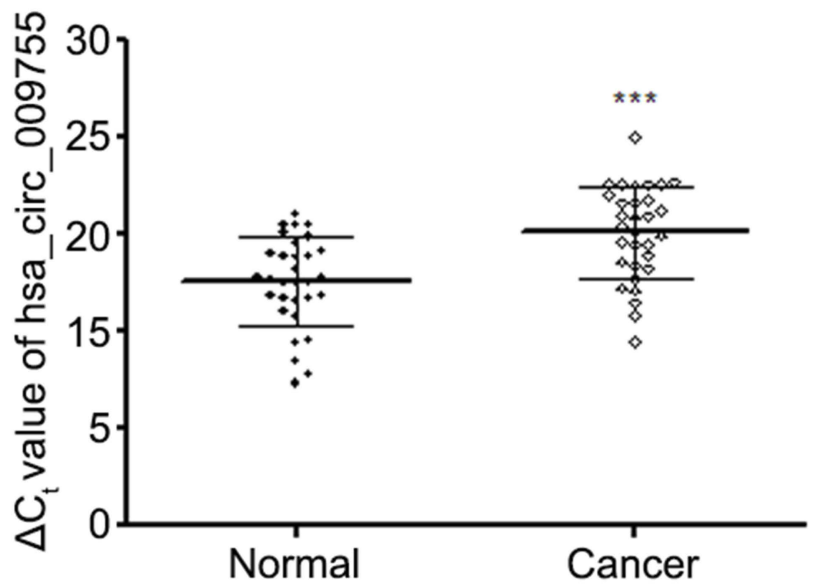

Figure 3 Low-level expression hsa_circ_009755 in OSCC tissues. Expression levels of hsa_circ_009755 in 27 pairs of OSCC tissues are shown relative to the levels in paired adjacent normal tissues. Higher $\Delta \mathrm{Ct}$ values indicate lower expression. Data are expressed as means $\pm \mathrm{SD} ; * * * P<0.001$.

Abbreviation: OSCC, oral squamous cell carcinoma.

OSCC tissues from paired adjacent normal tissues. The area under the ROC curve (AUC) was 0.782 (Figure 5A), the cutoff value was 13.22 (Figure 5B), and the corresponding sensitivity and specificity were $70.37 \%$ and $77.78 \%$, respectively. The ROC is used to reflect the sensitivity and specificity of continuous variables; the diagnostic value of the variables is high only when the AUC is large. Values below and above the cutoff value are negative and positive values, respectively, and the cutoff value is a schematic combination of sensitivity and specificity. Therefore, the calculated values suggest that hsa_circ_009755 could serve as a potential biomarker for the diagnosis of OSCC.

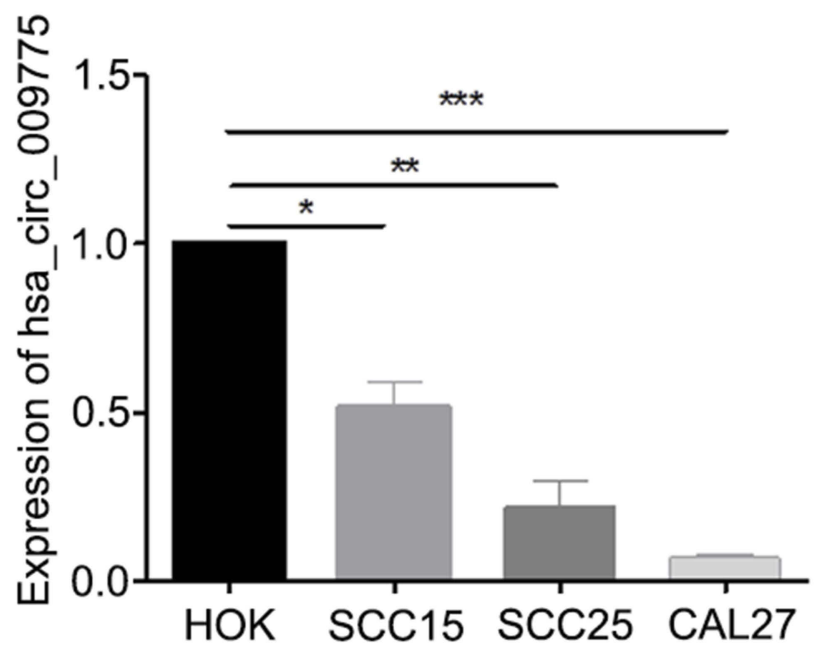

Figure 4 Expression of hsa_circ_009755 in distinct OSCC cell lines. Expression levels of hsa_circ_009755 in 3 OSCC cell lines (SCCI5, SCC25, and CAL27) and normal HOKcells were assessed using qRT-PCR. Data are presented as means $\pm S D ; * P<0.01, * * P<0.01, * * * P<0.001$.

Abbreviations: HOK, human oral keratinocyte; OSCC, oral squamous cell carcinoma; qRT-PCR, quantitative RT-PCR.

\section{Prediction of the circRNA/miRNA/ mRNA interaction}

In order to explore the molecular mechanism and function of circRNA hsa_circ_009755, we investigated the potential miRNAs and mRNAs bound to hsa_circ_009755. As shown in Figure 6, hsa_circ_009755 in cancer tissues may interact with has-miR-585-5p, has-miR-29a-5p, and has-miR-6079.

\section{Discussion}

Detection of circRNAs in RNA viruses was reported as early as in the $1970 \mathrm{~s},{ }^{11,12}$ and isolated reports of circRNA expression 
Table I Relationship between hsa_circ_009755 expression levels $(\Delta \mathrm{Ct})$ in cancer tissue and clinicopathological features of OSCC patients

\begin{tabular}{|c|c|c|c|}
\hline Characteristic & $\begin{array}{c}\text { Number } \\
\text { of } \\
\text { patients }\end{array}$ & $\begin{array}{c}\text { Mean } \pm \\
\text { SD }\end{array}$ & $P$-value \\
\hline \multicolumn{4}{|l|}{ Gender } \\
\hline Male & 21 & $15.15 \pm 2.37$ & 0.267 \\
\hline Female & 6 & $13.85 \pm 2.77$ & \\
\hline \multicolumn{4}{|l|}{ Age } \\
\hline$<60$ & 20 & $|4.6| \pm 2.65$ & 0.376 \\
\hline$\geq 60$ & 7 & $15.59 \pm 1.84$ & \\
\hline \multicolumn{4}{|l|}{ Tumor site } \\
\hline Tongue & 18 & $14.50 \pm 2.74$ & 0.565 \\
\hline Buccal & 3 & $15.89 \pm 1.50$ & \\
\hline Others & 6 & $15.43 \pm 1.97$ & \\
\hline \multicolumn{4}{|l|}{ T stage } \\
\hline $\mathrm{T}_{1}-\mathrm{T}_{2}$ & 13 & $13.64 \pm 2.37$ & $0.011 *$ \\
\hline $\mathrm{T}_{3}-\mathrm{T}_{4}$ & 14 & $15.99 \pm 2.04$ & \\
\hline \multicolumn{4}{|l|}{ Lymph node metastasis } \\
\hline $\mathrm{N}_{0}$ & 19 & $|5.4| \pm 2.13$ & 0.074 \\
\hline $\mathrm{N}_{1}-\mathrm{N}_{2}$ & 8 & $13.56 \pm 2.85$ & \\
\hline \multicolumn{4}{|l|}{ Differentiation } \\
\hline Well & 22 & $14.69 \pm 2.58$ & 0.456 \\
\hline Moderate and poor & 5 & $15.62 \pm 1.96$ & \\
\hline
\end{tabular}

Note: *Statistically significant.

Abbreviation: OSCC, oral squamous cell carcinoma.

from single genes were published in the subsequent decades; however, because of technological and methodological biases, the detected circRNAs were generally regarded as rare splicing "mistakes" until recently. ${ }^{4}$ Currently, circRNAs are regarded as abundant, stable, conserved, and nonrandom products of RNA splicing that could be involved in the control of gene expression. ${ }^{5}$ In accordance with previous work, we noted that the abnormal expression of circRNAs might be correlated with disease. For instance, circRNAs can interact with RNAbinding proteins or miRNAs and thereby influence the generation and development of cancer. ${ }^{13,14}$ Moreover, several types of tumor markers have been investigated, and the expression circRNAs has been reported to differ substantially between tumor cells and normal cells. ${ }^{6,15-20}$ These studies indicate that circRNAs could serve as potential cancer biomarkers. ${ }^{8,21,22}$

$\mathrm{Li}$ et al found that circITCH was markedly downregulated in esophageal squamous cell carcinoma samples; circITCH harbors sites for concurrently binding several miRNAs, which indicates that this circRNA could function as a cavernous transporter or regulator of miRNAs, and
A

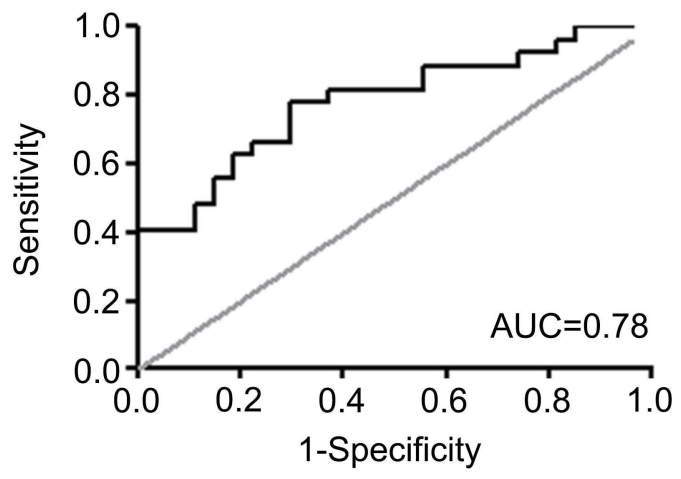

B

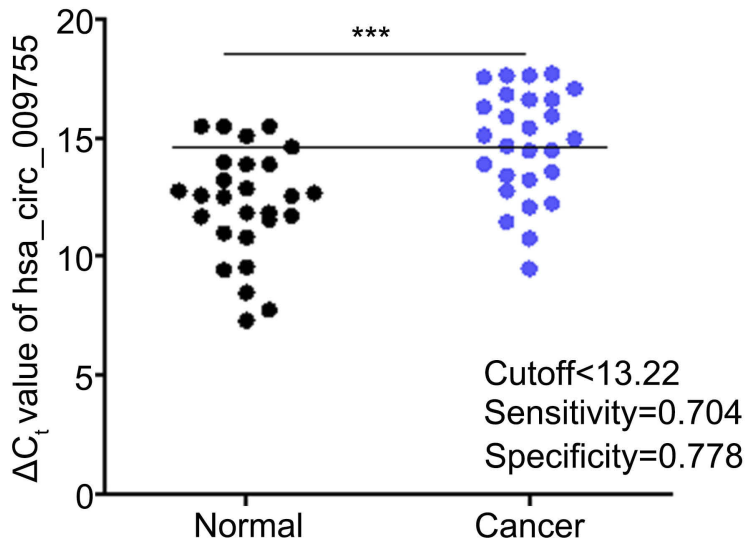

Figure 5 Diagnostic value of hsa_circ_009755 in OSCC.

Notes: (A) Area under the ROC curve was $0.78(95 \% \mathrm{Cl}=0.6670-0.8544$; $P<0.000 \mathrm{I}$ ), and (B) cutoff of hsa_circ_009755 was I3.22. Sensitivity and specificity were 0.704 and 0.778 , respectively. Data are presented as means $\pm S D ; * * * P<0.001$. Abbreviations: AUC, area under the ROC curve; OSCC, oral squamous cell carcinoma; ROC, receiver operating characteristic.

circITCH might inhibit tumor growth by regulating the Wnt/ $\beta$-catenin signaling pathway. ${ }^{8}$ Another circRNA reported to be associated with cell proliferation is circFOXO3; intriguingly, as in the case of circITCH, low levels of circFOXO3 appear to be associated with increased cell proliferation. ${ }^{23}$ Last, circHIPK3 is strongly downregulated in bladder cancer tissues and cell lines, and circHIPK3 overexpression effectively inhibits migration, invasion, and angiogenesis of bladder cancer cells in vitro and suppresses bladder cancer growth and metastasis in vivo; circHIPK3 contains two key binding sites for the miRNA miR-558 and can sponge large amounts of miR-558 and thereby suppress heparanase expression. ${ }^{24}$

The circRNA hsa_circ_009755 is located at chromosome 6q14.1 on the plus strand, is aligned in a sense orientation to the known protein-coding gene $V W A 8$, and spans exons 


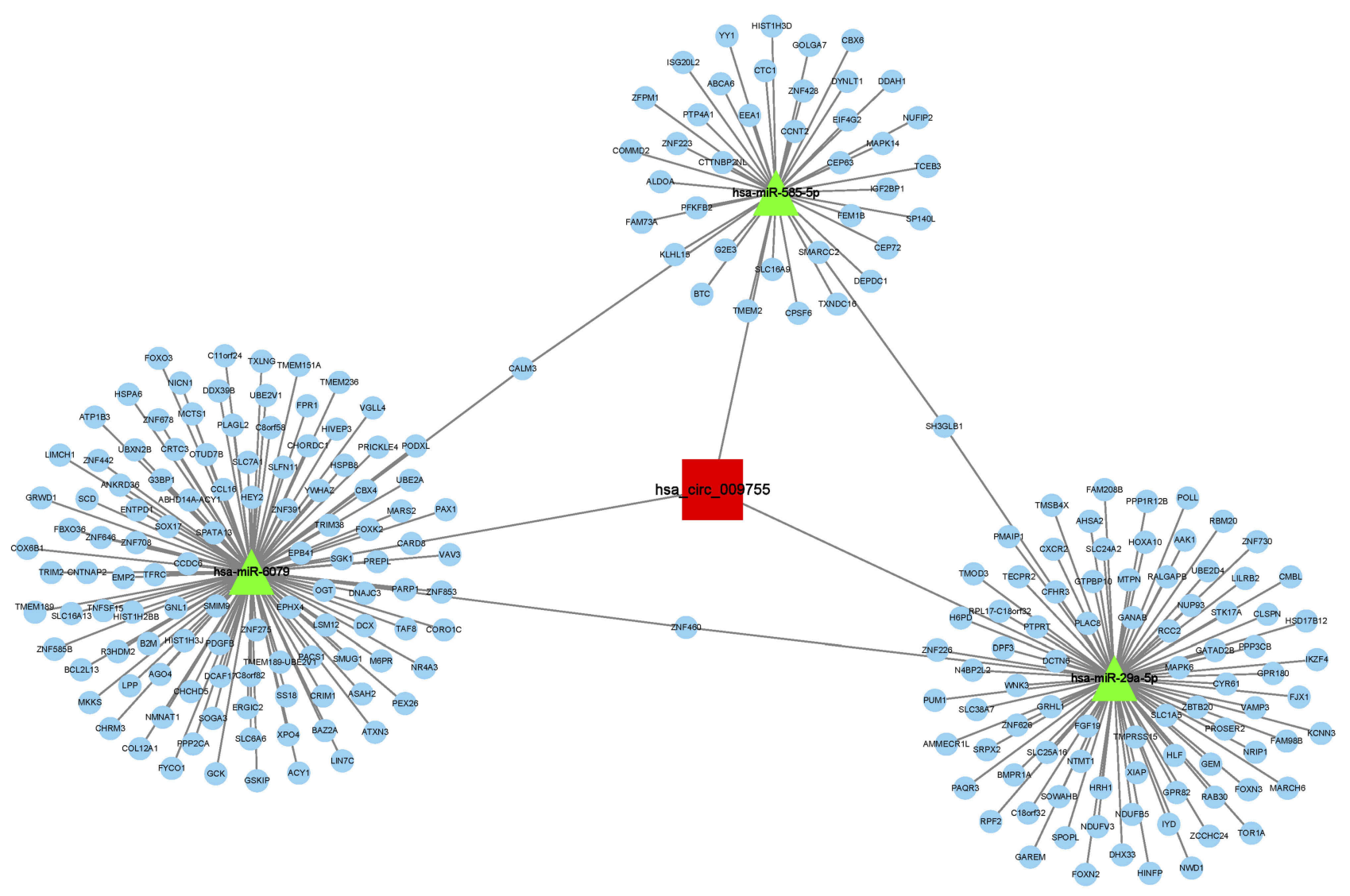

Figure 6 CircRNA-miRNA-mRNA network of hsa_circ_009755.

Notes: CircRNA: rectangular; miRNA: triangular; mRNA: circular.

13-16; however, the function of this circRNA in tumor progression has remained unknown. Here, we used TaqManbased RT-PCR analyses to compare hsa_circ_009755 expression levels in OSCC tissues and cell lines with those in normal tissues and cells, and our results showed that hsa_circ_009755 was drastically downregulated in OSCC; thus, hsa_circ_009755 expression might specifically and sensitively indicate the degree of OSCC. Notably, comparison of hsa_circ_009755 expression with clinicopathological characteristics of patients revealed that the circRNA expression level was significantly associated with the T stage. Hsa_circ_009755 might serve as a potential diagnostic biomarker for OSCC.

CircRNA plays an important role in regulating gene expression. It may be used as a gene sponge, an RBP sponge, or a transcription/translation regulator. ${ }^{25-27} \mathrm{We}$ found that hsa_circ_9755 was abnormal low expression in cancer tissues. It is predicted to be related to has-miR -585-5p, has-miR-29a-5p, and has-miR-6079. Our future research plan is to further explain the important role of hsa_circ_009755 in OSCC to unravel the molecular mechanisms of hsa_circ_9755 in OSCC and other cancers.

\section{Acknowledgments}

This study was supported by the National Natural Science Foundation of China (Grant no. 81572654), the Basic Research Program of Shenzhen Innovation Council of China (Grant nos. JCYJ20160428173933559, JCYJ20150403091443286 and SZBC2017023), and Sanming Project of Medicine in Shenzhen (SZSM 201512036, Oral and Maxillofacial Surgery Team, Professor Yu Guangyan, Stomatology Hospital Peking University).

\section{Disclosure}

The authors report no conflicts of interest in this work.

\section{References}

1. Lingen MW, Kalmar JR, Karrison T, Speight PM. Critical evaluation of diagnostic aids for the detection of oral cancer. Oral Oncol. 2008;44 (1):10-22. doi:10.1016/j.oraloncology.2007.06.011

2. Colevas AD, Yom SS, Pfister DG, et al. NCCN guidelines insights: head and neck cancers, version 1.2018. J Natl Compr Canc Netw. 2018;16(5):479-490. doi:10.6004/jnccn.2018.0026 
3. Rhodus NL, Kerr AR, Patel K. Oral cancer: leukoplakia, premalignancy, and squamous cell carcinoma. Dent Clin North Am. 2014;58(2):315-340. doi:10.1016/j.cden.2013.12.004

4. Salzman J, Gawad C, Wang PL, Lacayo N, Brown PO. Circular RNAs are the predominant transcript isoform from hundreds of human genes in diverse cell types. Preiss T, ed. PLoS One. 2012;7 (2):e30733. doi:10.1371/journal.pone.0030733

5. Jeck WR, Sorrentino JA, Wang K, et al. Circular RNAs are abundant, conserved, and associated with ALU repeats. RNA. 2012;19:141-157. doi:10.1261/rna.035667.112

6. Liang H-F, Zhang X-Z, Liu B-G, Jia G-T, Li W-L. Circular RNA circ-ABCB10 promotes breast cancer proliferation and progression through sponging miR-1271. Am J Cancer Res. 2017;7 (7):1566-1576.

7. Zhu M, Xu Y, Chen Y, Yan F. Circular BANP, an upregulated circular RNA that modulates cell proliferation in colorectal cancer. Biomed Pharmacother. 2017;88:138-144. doi:10.1016/j.biopha.2016.12.097

8. Li F, Zhang L, Li W, et al. Circular RNA ITCH has inhibitory effect on ESCC by suppressing the Wnt/ $\beta$-catenin pathway. Oncotarget. 2015;6(8):6001-6013.

9. Guo W, Zhang J, Zhang D, et al. Polymorphisms and expression pattern of circular RNA circ-ITCH contributes to the carcinogenesis of hepatocellular carcinoma. Oncotarget. 2017;8(29):48169-48177.

10. Song H, Sun W, Ye G, et al. Long non-coding RNA expression profile in human gastric cancer and its clinical significances. $J$ Transl Med. 2013;11:225. doi:10.1186/1479-5876-11-225

11. Wilusz JE, Sharp PA. Molecular biology. A circuitous route to noncoding RNA. Science. 2013;340(6131):440-441. doi:10.1126/ science. 1238522

12. Wu Q, Wang Y, Cao M, et al. Homology-independent discovery of replicating pathogenic circular RNAs by deep sequencing and a new computational algorithm. Proc Natl Acad Sci. 2012;109 (10):3938-3943. doi:10.1073/pnas.1117815109

13. Zhang Y, Liu H, Li W, et al. CircRNA_100269 is downregulated in gastric cancer and suppresses tumor cell growth by targeting miR-630. Aging (Albany NY). 2017;9(6):1585-1594. doi:10.18632/ aging.101254

14. Zhang H, Wang G, Ding C, et al. Increased circular RNA UBAP2 acts as a sponge of miR-143 to promote osteosarcoma progression. Oncotarget. 2017;8(37):61687-61697. doi:10.18632/oncotarget.18671
15. Xia W, Qiu M, Chen R, et al. Circular RNA has circ 0067934 is upregulated in esophageal squamous cell carcinoma and promoted proliferation. Sci Rep. 2016;6(1). doi:10.1038/srep35576.

16. Yang P, Qiu Z, Jiang Y, et al. Silencing of cZNF292 circular RNA suppresses human glioma tube formation via the Wnt/ $\beta$-catenin signaling pathway. Oncotarget. 2016;7(39):63449-63455.

17. Zhu X, Wang X, Wei S, et al. hsa_circ_0013958: a circular RNA and potential novel biomarker for lung adenocarcinoma. FEBS J. 2017;284(14):2170-2182. doi:10.1111/febs. 14132

18. Chen L, Zhang S, Wu J, et al. circRNA_100290 plays a role in oral cancer by functioning as a sponge of the miR-29 family. Oncogene. 2017;36(32):4551-4561. doi:10.1038/onc.2017.89

19. Kong Z, Wan X, Zhang Y, et al. Androgen-responsive circular RNA circSMARCA5 is up-regulated and promotes cell proliferation in prostate cancer. Biochem Biophys Res Commun. 2017;493:1217-1223. doi:10.1016/j.bbrc.2017.07.162

20. Chen -L-L, Yang L. Regulation of circRNA biogenesis. RNA Biol. 2015;12(4):381-388. doi:10.1080/15476286.2015.1020271

21. Chen Y, Li C, Tan C, Liu X. Circular RNAs: a new frontier in the study of human diseases. J Med Genet. 2016;53(6):359-365. doi:10.1136/jmedgenet-2016-103758

22. Meng S, Zhou H, Feng Z, et al. CircRNA: functions and properties of a novel potential biomarker for cancer. Mol Cancer. 2017;16(1):94. doi:10.1186/s12943-017-0663-2

23. Du WW, Yang W, Liu E, Yang Z, Dhaliwal P, Yang BB. Foxo3 circular RNA retards cell cycle progression via forming ternary complexes with p21 and CDK2. Nucleic Acids Res. 2016;44 (6):2846-2858. doi:10.1093/nar/gkw027

24. Li Y, Zheng F, Xiao X, et al. CircHIPK3 sponges miR-558 to suppress heparanase expression in bladder cancer cells. EMBO Rep. 2017;18(9):1646-1659. doi:10.15252/embr.201643581

25. Li Z, Huang C, Bao C, et al. Exon-intron circular RNAs regulate transcription in the nucleus. Nat Struct Mol Biol. 2015;22 (3):256-264. doi:10.1038/nsmb.2959

26. Hansen TB, Jensen TI, Clausen BH, et al. Natural RNA circles function as efficient microRNA sponges. Nature. 2013;495 (7441):384-388. doi:10.1038/nature11993

27. Hentze MW, Preiss T. Circular RNAs: splicing's enigma variations. Embo J. 2013;32(7):923-925. doi:10.1038/emboj.2013.53
OncoTargets and Therapy

\section{Publish your work in this journal}

OncoTargets and Therapy is an international, peer-reviewed, open access journal focusing on the pathological basis of all cancers, potential targets for therapy and treatment protocols employed to improve the management of cancer patients. The journal also focuses on the impact of management programs and new therapeutic agents and protocols on patient perspectives such as quality of life, adherence and satisfaction. The manuscript management system is completely online and includes a very quick and fair peer-review system, which is all easy to use. Visit http://www.dovepress.com/ testimonials.php to read real quotes from published authors. 